\title{
Let us not discard a preexisting epidural catheter for intrapartum cesarean section yet!
}

\author{
Anju Romina Bhalotra \\ Department of Anesthesiology, Maulana Azad Medical College and Lok Nayak Hospital, New Delhi, India
}

I read with interest the study by Yoon et al. [1] comparing epidural surgical anesthesia (ESA) and spinal anesthesia (SA) following epidural labor analgesia (ELA) for intrapartum cesarean section (CS). I also read the accompanying editorial by Kim [2] in which the author raises the question whether a pre-existing epidural catheter should be discarded for an intrapartum CS. Yoon et al. [1] suggest that ESA for CS has been unsuccessful or fails to achieve a satisfactory block in approximately $1.7-38 \%$ of cases, depending upon how failure is defined. They compared the rate of pain-free intrapartum CS performed under ESA and SA after ELA. While the failure rate in achieving pain-free surgery was significantly higher in the ESA group than in the SA group $(15.3 \%$ vs. $2.5 \%, \mathrm{P}<0.001)$, there was no statistically significant difference between groups in the rate of conversion to general anesthesia. Yoon et al. [1] excluded patients with less than a 2-hour interval between epidural analgesia top-ups and CS in their study. This implies that when the patients were taken up for CS, the epidural analgesia may have worn off to a large extent. The pre-existing level of sensory block at the start of CS was T10 on assessment by loss of sensation to cold and T12 and L1 respectively, on assessment by pinprick in both the groups. Thus it is likely that the intensity of block was less in the ESA group, making this study more of a comparison of epidural vs.

Corresponding author: Anju Romina Bhalotra, M.D.

Department of Anesthesiology, Maulana Azad Medical College and Lok Nayak Hospital, A-1/59 Safdarjang Enclave, New Delhi 110029, India

Tel: 01126183684, Fax: 01126183684

Email:drakgk@yahoo.co.in

ORCID: https://orcid.org/0000-0003-0700-2941

Received: September 17, 2017.

Accepted: September 29, 2017.

Korean J Anesthesiol 2018 June 71(3): 244-245

https://doi.org/10.4097/kja.d.17.00017
SA for CS rather than extension of ELA to ESA. The use of epidural anesthesia for elective CS is less common, as the resulting block is less reliable than that with SA. Besides, the combined spinal-epidural (CSE) technique offers advantages of rapid onset and the ability to augment or prolong anesthesia as desired.

A T10 level of epidural analgesia needs to be extended to T4 level to enable optimal anesthesia to perform CS. This typically requires a volume of 15 to $20 \mathrm{ml}$ of local anesthetic with one or more adjuvants. A fractionated dosing schedule may be used to offer greater hemodynamic stability; this allows assessment of the evolving sensory level prior to administration of the full dose of local anesthetic and minimizes dural sac compression. Besides, it allows early sensory blockade at the incision site, so that surgery can proceed without delay in emergency cases, prior to the establishment of a full T4 level block [3].

In order to reduce the failure rate of an epidural block, meticulous attention must be paid to technical details and a combination of local anesthetic and opioid should be used. Besides, it is important to understand the different characteristics of epidural vs. spinal blockade. It should be explained to the patient that while a sensation of deep pressure and movement may be felt, any discomfort or pain will be addressed promptly. In a review by Lee et al. [4], the predictors of failed ELA for CS included initiation of labor analgesia with plain epidural technique (compared to CSE), two or more episodes of breakthrough pain during labor, and a prolonged duration of neuraxial labor analgesia. Epidural catheters in situ for a longer duration were at higher risk of migration. They suggest that frequent use of CSE analgesia, early replacement of "uncertain" catheters during labor analgesia, and the experience of anesthesia providers may have contributed to their high success rate.

Neuraxial anesthesia is preferred wherever possible as the preferred method of providing anesthesia for CS. This is due to the increasing use of epidural techniques for labor analgesia and the ease of rapid augmentation of the block to provide anesthe-

(c) This is an open-access article distributed under the terms of the Creative Commons Attribution Non-Commercial License (http://creativecommons.org/ licenses/by-nc/4.0/), which permits unrestricted non-commercial use, distribution, and reproduction in any medium, provided the original work is properly cited. 
sia if an emergency CS is required. Besides, there is heightened awareness of the risks of airway complications during general anesthesia in pregnant patients, improvement in the quality of neuraxial anesthesia using a combination of local anesthetics and opioids, limited transplacental drug transfer, and the ability of the mother to remain awake to experience childbirth. General anesthesia for CS is associated with a higher maternal mortality, especially if carried out in an emergency situation. Furthermore, administration of a standard intrathecal dose of bupivacaine in the setting of a partial but failed epidural may result in a high spinal block. Radiographic evidence suggests that the dural sac may be compressed by prior epidural drug administration [5]. Yoon et al. [1] noted a significantly higher mean phenylephrine requirement in the SA group compared to ELA group $(\mathrm{P}<0.001)$. Thus, when performing SA after a failed epidural or spinal anesthesia, reducing the dose of bupivacaine depending on the extent of the existing blockade should be considered as there is a risk of a high spinal block with associated complications. There are several important reasons to optimize the use of a preexisting epidural catheter and extend ELA to ESA, rather than consider abandoning the use of this catheter as a technique to provide intrapartum anesthesia for CS.

\section{References}

1. Yoon HJ, Do SH, Yun YJ. Comparing epidural surgical anesthesia and spinal anesthesia following epidural labor analgesia for intrapartum cesarean section: a prospective randomized controlled trial. Korean J Anesthesiol 2017; 70: 412-9.

2. Kim DK. Anything that can go wrong, will go wrong: should a pre-existing epidural catheter be discarded for an intrapartum cesarean section? Korean J Anesthesiol 2017; 70: 373-4.

3. Lawrence CS. Anesthesia for cesarean delivery. In: Chestnut's Obstetric Anesthesia. 4th ed. Edited by Chestnut DH, Polley LS, Tsen LS, Wong CA: Philadelphia, Elsevier. 2009, p 543.

4. Lee S, Lew E, Lim Y, Sia AT. Failure of augmentation of labor epidural analgesia for intrapartum cesarean delivery: a retrospective review. Anesth Analg 2009; 108: 252-4.

5. Higuchi H, Adachi Y, Kazama T. Effects of epidural saline injection on cerebrospinal fluid volume and velocity waveform: a magnetic resonance imaging study. Anesthesiology 2005; 102: 285-92. 